\title{
Debate: \\ What Is Optimal First-Line Therapy for Chronic Lymphocytic Leukemia?
}

Presented by Mazyar Shadman, MD, MPH, and Deborah M. Stephens, DO

\section{ABSTRACT}

In the era of newer, highly effective targeted therapies for chronic lymphocytic leukemia (CLL), experts debated whether there is still a role for chemoimmunotherapy in the first-line setting for the treatment of this disease. In general, targeted therapies are preferred as first-line treatment for all patients, with the exception of low-risk patients [younger, fit patients with mutated IGHV who are candidates for fludarabine/cyclophosphamide/rituximab (FCR)]. About $37 \%$ of low-risk patients experience long-term durable remissions and remain stable after treatment with FCR for many years. Advantages of FCR over small molecule inhibitors include cost, fixed duration treatment, and long-term survival benefit. Disadvantages are an etimated $5 \%$ risk of developing myelodysplastic syndrome or acute myeloid leukemia. Advantages of small molecule inhibitors are improvements in progression-free and overall survival and quality of life when compared with chemoimmunotherapy. Disadvantages of small molecule inhibitors are high cost and the need for continuous treatment. However, studies are underway to develop fixed duration regimens with combinations of small molecule inhibitors that aim to deepen remissions.

J Natl Compr Canc Netw 2020;18(7.5):993-997 doi: 10.6004/jnccn.2020.5011

Over the past 6 years, the treatment armamentarium for chronic lymphocytic leukemia (CLL) has expanded from chemoimmunotherapy to include novel small molecule inhibitors, such as ibrutinib, venetoclax, and acalabrutinib. In the current era, with several new therapies available to treat CLL, the question on many oncologists' minds is whether chemoimmunotherapy still has a role as first-line treatment.

At the NCCN 2020 Virtual Annual Conference, 2 experts debated that question. Mazyar Shadman, MD, MPH, Assistant Professor, Fred Hutchinson Cancer Research Center/University of Washington, took the position that "Chemotherapy is Not Dead as First-Line Treatment of CLL," and Deborah M. Stephens, DO, Assistant Professor, Huntsman Cancer Center at the University of Utah, spoke in support of novel therapies as first line treatment. The debate was followed by expert discussion (see sidebar) from Andrew D. Zelenetz, MD, PhD, Professor of Medicine, Memorial Sloan Kettering Cancer Center; Julio C. Chavez, MD, Assistant Professor, Moffitt Cancer Center; and Shuo Ma, MD, PhD, Associate Professor of Medicine, Robert H. Lurie Comprehensive Cancer Center of Northwestern University.

\section{Chemotherapy Is Not Dead as First-Line Treatment of CLL}

"This is an exciting time for CLL, and we have a number of novel agents that include ibrutinib, acalabrutinib, venetoclax, duvelisib, idelalisib, obinutuzumab and ofatumumab, many of which can be used in the firstline setting," Dr. Shadman stated. He pointed out that his remarks did not apply to CLL with abnormal TP53. "There is no role for chemoimmunotherapy for these patients. The standard of care for abnormal TP53 is novel agents."

Chemoimmunotherapy entails fixed duration treatment, whereas some novel agents are currently given continuously until disease progression. Chemoimmunotherapy also shows high rates of disease-free survival and durable remissions, Dr. Shadman continued. Other advantages of chemoimmunotherapy include more widespread access and lower costs of treatment compared with novel targeted agents.

"In the United States, access to novel drugs is an issue. We need to better identify a specific population that can benefit from chemoimmunotherapy," he said.

Among chemoimmunotherapy regimens, fludarabine/ cyclophosphamide/rituximab (FCR) has the longest follow-up in the first-line setting and improves progressionfree survival (PFS) compared with bendamustine/rituximab (BR) and both PFS and overall survival (OS) compared with fludarabine/cyclophosphamide (FC). Chlorambucil/ obinutuzumab is another regimen that improves OS compared with chlorambucil/rituximab.

"Chemoimmunotherapy regimens are selected for frontline treatment based on fitness, age, and comorbidities," Dr. Shadman said. 
Chronic Lymphocytic Leukemia: Expert Opinion

After the debate, additional experts discussed some of the finer points of the evidence: Andrew D. Zelenetz, MD, PhD, Memorial Sloan Kettering Cancer Center; Julio C. Chavez, MD, Moffitt Cancer Center; and Shuo Ma, MD, PhD, Robert H. Lurie Comprehensive Cancer Center of Northwestern University.

Basically, all 3 experts agreed that they follow what clinical trials show, but noted subtleties or nuances of the trials and unanswered questions. According to Dr. Ma, "The discussion has evolved over the past several years, since there have been quite a few randomized trials on small molecule inhibitors versus chemoimmunotherapy. Based on those data, my discussion with patients is shifting. We all agree there is not much of a role for chemoimmunotherapy in patients with a TP53 mutation. For other patients, we look at IGHV status. For unmutated IGHV $\mathrm{CLL}$, targeted treatment therapies outperform chemoimmunotherapy."

All 3 experts agreed that for young, fit patients with mutated IGHV, there is still a role for FCR, and that has to be part of the discussion. That being said, "these patients do as well on small molecule inhibitors," Dr. Ma said.

"There are subtleties that aren't considered in randomized controlled trials. Trials don't measure things that are important to consider, such as quality of life, long-term side effects, and cost, as well as patient's preferences, which is a main driver of treatment selection. A patient may prefer to have a fixed duration of treatment, for example," Dr. Chavez noted.

"There is no question that chemoimmunotherapy is dead for the patient who has TP53, but del 17p is important. We sequence $100 \%$ of our patients for the TP53 mutation, because we can see those mutations without $17 p$. There is little controversy that the best treatment for those patients is targeted therapy," Dr. Zelenetz stated. "I use IGHV as a critical determinant in low-risk patients," he continued.

Dr. Ma said that she uses IGHV status to help guide therapy only for young, fit patients who may be potential candidates for chemoimmunotherapy. "IGHV status will inform treatment. The recommendation is to test for IGHV for prognostic purposes and for predicting treatment effect. It makes some difference," she said.

Dr. Chavez agreed, noting that about $37 \%$ of low-risk patients do not experience relapse at 10 years after treatment with FCR. "I have treated several of these patients younger than 60 with FCR, and some achieved long-term remissions. However, this is a very select group of patients. We are using less and less chemoimmunotherapy. We need to use this approach when indicated and in the right setting as it is not an easy regimen to administer," Dr. Chavez said. "And I point out to patients that FCR is cheaper but potentially more toxic than venetoclax."

Dr. Zelenetz pointed out that treatment-free intervals make patients feel psychologically better. Other issues to be considered, he added, were financial toxicity with continuous treatment and identifying the best sequence.

"We know we can use targeted therapy after chemoimmunotherapy failure, but we don't know if patients who fail

(continued)
In the frontline setting, ibrutinib has been shown to improve OS compared to FCR, but not for CLL with mutated $I G H V$. Ibrutinib also improves PFS compared with other chemoimmunotherapy regimens, namely bendamustine and rituximab. Both venetoclax and acalabrutinib improve PFS compared with chlorambucil (in combination with obinutuzumab). However, there is no head-to-head trial indicating superiority of one of the novel agents over others.

"Before 2018, we used to have long discussions with patients who require frontline treatment about the pros and cons of chemoimmunotherapy versus ibrutinib," he said.

After FCR, the risk of treatment-related myelodysplastic syndrome (MDS) or acute myeloid leukemia (AML) is $5 \%$. However, patients with mutated $I G H V$ have durable long-term remissions from FCR. "It makes sense for some patients to accept the potential risks of FCR," Dr. Shadman said. "Before 2018, most experts favored chemoimmunotherapy for patients with mutated $I G H V$."

Several studies paved the way for use of novel agents as frontline therapy. These include ECOG 1912, the ALLIANCE trial (A041202), and the CLL14 study. ${ }^{2-5}$ ECOG 2912 compared FCR versus ibrutinib/rituximab as frontline treatment in 529 younger, fit patients with CLL without the $17 \mathrm{p}$ deletion. No crossover was allowed. ${ }^{2}$ At a median follow-up of 33.6 months, ibrutinib/rituximab had a significant benefit over FCR in PFS $(89.4 \%$ vs $72.9 \%$, respectively; $P<.001)$ and OS (98.8\% vs $91.5 \%$, respectively; $P=.001)$. The benefit of ibrutinib/rituximab was driven by unmutated IGHV CLL, but no significant difference was seen in PFS for mutated IGHV. These results held up in a longer-term analysis at 48 months. ${ }^{3}$

"This is an important study that included all comers (mutated and unmutated IGHV) and met the [OS] endpoint," Dr. Shadman said.

The Alliance trial (A041202) enrolled older patients with untreated CLL and compared bendamustine/rituximab versus ibrutinib plus or minus rituximab. ${ }^{4}$ The ibrutinibcontaining regimens had a PFS benefit but no OS benefit compared with bendamustine/rituximab. IGHV mutational status did not significantly change the difference in PFS in different arms. The improved PFS with ibrutinib in patients with a mutated IGHV became evident later in treatment. ${ }^{4}$

The CLL14 study enrolled 432 older patients with comorbidities and randomized them to receive venetoclax/ obinutuzumab versus chlorambucil/obinutuzumab as front-line therapy. ${ }^{5}$ Patients with unmutated $I G H V$ had significantly improved PFS on venetoclax/obinutuzumab at 24 months ( $88.2 \%$ vs $64.1 \%$, respectively), but no significant difference was seen for patients with TP53 or $I G H V$ mutations for either regimen at that time point. In fact, patients with mutated IGHV CLL did well with chlorambucil/obinutuzumab, and longer follow-up at a median of 39.6 months showed sustained benefit. ${ }^{6}$ 
Chronic Lymphocytic Leukemia: Expert Opinion (cont.)

on targeted therapy should get chemoimmunotherapy. We may not need to consider that because now there are better targeted therapies," Dr. Zelenetz said.

"With small molecule inhibitors, we currently do not have enough data to know what's the best sequencing. Nowadays, most of my discussion with patients is which targeted therapy to choose as first-line treatment-BTK inhibitors versus BCL2 inhibitors. It's a matter of considering patient factors such as comorbidities, renal function, and patient preferences. Some patients don't want to have a treatment that involves frequent clinic visits to monitor for tumor lysis syndrome. Older patients typically want an easy start with BTK inhibitors without intensive monitoring," Dr. Ma said. "On the other hand, many patients love time-limited combination therapy with venetoclax."

Regarding minimal residual disease (MRD) testing, the current standard is flow cytometry, but molecular assays are being developed that might make it easier to test in the community with a reproducible technique. "How are you using MRD in your practice? At what point would you stop therapy?" Dr. Zelenetz asked the other 2 experts.

"There is not much data to guide us on the optimal duration of treatment. Not all patients achieve MRD-undetectable status at the end of a fixed duration therapy. I don't think we know if MRD-positive patients benefit from continuous venetoclax treatment," Dr. Ma said. "We do know that a small portion of patients can convert from MRD-detectable to undetectable on continuing venetoclax. After 12 months of treatment [with venetoclax and obinutuzumab], I test for MRD by flow cytometry. If there is detectable MRD, I would discuss with the patient another 6 months of venetoclax and repeat testing to see if their MRD has converted," she continued.

"We know that progression-free survival correlates with depth of response, and undetectable MRD translates to a much longer progression-free survival. This is an argument to try to achieve undetectable MRD, especially for first-line treatment," she finished.

"I also check with MRD by flow cytometry, and if there is detectable MRD at 12 months, it is probably best to continue on therapy in CLL 114-like patients. If there is $17 p$ deletion, I tend to treat longer with venetoclax," Dr. Chavez said.

"There are 2 groups of patients detected by MRD testing; one group steadily declines over time and a second group declines and then reaccumulates MRD. I don't know what to do about these 2 different populations. I check MRD throughout the treatment, usually testing quarterly to get a sense of the trajectory of each patient. I think this will help me at the end of the year to decide what to do. More data are better than no data," Dr. Zelenetz said. "Next generation sequencing assays in development may be helpful to community oncologists who don't have access to flow cytometry for MRD testing," he added.

All 3 doctors agreed that the COVID-19 pandemic is shifting their thinking favoring small molecule inhibitors. "In this current situation we want to preserve immune function and try not to choose a therapy that is very immunosuppressive," Dr. Ma said.
"What we learned from these 3 head-to-head studies was that the primary endpoint was generally met in the unmutated $I G H V$ population but not in the $I G H V$ mutated group. The question is can we justify changing our practice based on these major trials," Dr. Shadman said. "In general, study results to date suggest that FCR can be considered for younger fit patients with mutated $I G H V$, and bendamustine/rituximab could be considered for older patients with mutated $I G H V$ if chemoimmunotherapy is desired. Consider chlorambucil/obinutuzumab for unfit or older patients with mutated IGHV if chemoimmunotherapy is desired," he continued.

"Patients need to be aware of the risk of secondary MDS/AML. Some patients won't want to take that risk. Others who believe that a $60 \%$ to $70 \%$ chance of 10 -year remission is possible with chemoimmunotherapy will want to take that $5 \%$ risk," Dr. Shadman said. "FCR is a reasonable option for previously untreated CLL in young, fit patients with mutated $I G H V$ without a $17 p$ deletion, but it is not a preferred choice. Targeted agents are always reasonable to consider, such as ibrutinib, acalabrutinib, and venetoclax," he stated. "Patients should be educated on the risks of secondary MDS/AML with FCR in order to make an informed choice."

Smaller studies suggest that adding a Burton's tyrosine kinase inhibitor (BTKi) can deepen the response to chemoimmunotherapy in patients without $\operatorname{del}(17 \mathrm{p})$ or TP53 mutation. In one study, ibrutinib was given for 1 week and then FCR was added for up to 6 cycles, followed by ibrutinib until disease progression or unacceptable toxicity. This small study found "the highest rate" of minimal residual disease (MRD) in bone marrow ever published in CLL without restricted prognostic marker status, according to the authors of that study. ${ }^{7}$

A second study in 45 patients with mutated IGHV found that ibrutinib given for 9 courses to all patients (after 3 courses of ibrutinib FCG), then obinutuzumab given for either 3 courses or 9 courses depending on MRD status in bone marrow could deepen response to chemoimmunotherapy over time, with "excellent progression-free survival and overall survival," Dr. Shadman said. ${ }^{8}$

The advantages of chemoimmunotherapy are fixed duration of treatment, lack of access to novel agents, and lower cost compared with novel agents, he reiterated. "FCR is a reasonable option for young fit patients with mutated IGHV and intact TP53. Targeted agents [ibrutinib, acalabrutinib, and venetoclax] are also reasonable in those patients," he stated.

"Time-limited and chemotherapy-free options (ie, venetoclax-based regimens) may change the landscape if longer follow-up shows durable treatment-free remissions and the drugs are affordable and accessible," Dr. Shadman concluded. 


\section{Small Molecule Inhibitors: Optimal First-Line Therapy for CLL}

Current choices for optimal frontline therapy of CLL include chemoimmunotherapy (FCR, bendamustine/rituximab [BR], chlorambucil/obinutuzumab ) or regimens that include small molecule inhibitors (ibrutinib with or without anti-CD20-monoclonal antibody, acalabrutinib with or without obinutuzumab, venetoclax/obinutuzumab). Deborah M. Stephens, DO, Huntsman Cancer Center of the University of Utah, cited several reasons to choose small molecule inhibitors over chemoimmunotherapy:

- prolonged PFS and OS

- survival benefit maintained in high-risk group (IGHV unmutated)

- possible deeper responses when used as earlier line of therapy

- improved quality of life

"I will focus on ibrutinib, because it has the longest follow-up data of all these agents," Dr. Stephens said.

The first study she talked about was ECOG 1912, which was also discussed by Dr. Shadman. ${ }^{2}$ ECOG 1912 randomized younger ( $<70$ years) fit patients to frontline treatment with ibrutinib/rituximab and FCR. Patients in the ibrutinib arm received continuous ibrutinib therapy combined with 6 cycles of rituximab, while patients in the FCR arm received fixed duration treatment of 6 cycles.

Extended follow-up at 48 months showed 3-year PFS rates that were "impressive," Dr. Stephens said: $89 \%$ for the ibrutinib arm versus $71 \%$ for FCR $(P<.0001) .{ }^{9}$ Additionally, these were the first data to show prolonged OS with ibrutinib/rituximab over FCR. Three-year OS rates were $99 \%$ and $93 \%$, respectively $(P=.009)$.

"It was also very exciting to see much better survival rates in the group of patients with unmutated $I G H V$, who were previously considered high risk and less likely to have prolonged survival," she continued. In patients with unmutated IGHV, 3-year OS rates were $89 \%$ and $65 \%$, respectively $(P<.0001)$, whereas the estimated survival was not that different in the group of patients with mutated IGHV: $89 \%$ versus $82 \%$, respectively.

"Despite the significant clinical efficacy of ibrutinib, only a limited number of patients achieve complete response [CR], which is why this agent has to be given continuously," Dr. Stephens explained.

The CR rate was $26 \%$ in a study of 327 patients (31 treatment-naïve) treated with ibrutinib. One of the only factors that increased chances of CR was no prior therapy, suggesting that earlier treatment can improve response. ${ }^{10} \mathrm{~A}$ second series of 208 patients (31 treatmentnaïve), showed that patients who experience CR on ibrutinib have prolonged PFS.9 "In summary, earlier treatment with ibrutinib can increase rates of CR, which can therefore prolong survival," Dr. Stephens stated.

"Small molecule inhibitors can improve quality of life," she continued. A good example is the RESONATE 2 trial, which showed that quality of life was improved with ibrutinib compared with chlorambucil on multiple quality of life questionnaires. Clinically meaningful improvements in multiple quality of life domains were maintained over time, as were improvement in disease-related symptoms." 11

Reasons not to choose chemoimmunotherapy as frontline treatment of CLL include the long-term risk of MDS/AML ( $5 \%$ at 12.8 years of follow-up), also cited by Dr. Shadman. ${ }^{1}$ A second reason is that chemoimmunotherapy poses the risk of inducing resistant mutations associated with chemorefractory disease. "FCR, the chemoimmunotherapy regimen with the longest follow-up, is known to select for TP53 resistant clones," she said.

"In choosing optimal frontline CLL therapy in 2020, don't be distracted by the toxicities of ibrutinib or the fact that ibrutinib and other small molecule inhibitors are mainly used as continuous therapy." Ibrutinib-related toxicities improve over time (except for hypertension, which is medically manageable). Importantly, the risk of infection with ibrutinib is also reduced over time. Acalabrutinib, approved for CLL in 2019, is a more selective BTKi than ibrutinib. It is designed to maintain efficacy but with reduced toxicity. ${ }^{12}$

The need for continuous therapy is considered a disadvantage of small molecule inhibitors, and efforts have been made to develop a regimen that can be given for a fixed duration. Currently, venetoclax and obintuzumab is a time-limited targeted regimen given for 12 months in the frontline setting, Dr. Stephens noted.

Several large ongoing trials are evaluating different approaches to administer ibrutinib as a fixed duration regimen. For example, in the multicohort phase II CAPTIVATE-MRD trial, 164 patients with previously untreated CLL were treated with ibrutinib for 3 months and then given 12 cycles of ibrutinib/venetoclax. This regime is an all-oral, chemotherapy-free regimen that leads to high rates of undetectable MRD in blood and bone marrow. ${ }^{13}$ After 12 months, patients were randomized to ibrutinib and placebo according to MRD status. However, results from the MRD-guided phase are not yet available.

"This will be a key study to determine which patients can discontinue and which patients need to continue ibrutinib," she said.

All 3 small molecule inhibitor regimens (ibrutinib with or without anti-CD20 MAB; acalabrutinib with or without obinutuzumab; or venetoclax plus obinutuzumab) prolong PFS and OS. Survival benefit is also observed in high-risk patients (ie, with unmutated $I G H V$ ). 
Using these regimens early may lead to deeper response and improved quality of life, Dr. Stephens said.

Disclosures: Dr. Shadman has disclosed that he has received grant/research support from AbbVie, Inc., Acerta Pharma, AstraZeneca Pharmaceuticals LP, BeiGene, Celgene Corporation, for Genentech, Inc., Gilead Sciences, Inc., Merck \& Co., Mustang Bio, Pharmacyclics, Sunesis Pharmaceuticals, Inc. and TG Therapeutics; is a scientific advisor for AstraZeneca, AbbVie, Inc. ADC Therapeutics, AstraZeneca Pharmaceuticals LP, Atara Biotherapeutics, Cellectar Biosciences, Genentech, Pharmacyclics, Sound Biologics, and Verastem. Dr. Stephens has disclosed that she has received grant/research support from Acerta Pharma, Gilead Sciences, Inc., Juno Therapeutics, Inc., mingSight, Arqule, and Karyopharm Therapeutics; and consulting fees from Janssen Pharmaceutical Products, Pharmacyclics, Karyopharm, and Innate. Dr. Chavez has disclosed that he has received grant/research support from Merck \& Co.; and consulting fees from Bayer HealthCare, BMS/Celgene, Karyopharm Therapeutics, Kite Pharma, MorphoSys AG, and Novartis Pharmaceuticals; and is on the Product/Speakers Bureau for AstraZeneca Pharmaceuticals LP, BeiGene, and Genentech, Inc.
Dr. Ma has disclosed that she has received grant/research support from AbbVie, Inc., AstraZeneca, BeiGene, Janssen Pharmaceutica Products, LP, Juno Therapeutics, Inc., Pharmacyclics, and TG Therapeutics; is a scientific advisor for AstraZeneca, Genentech, Inc. and Kite Pharma; and is on the Product/Speakers Bureau for AstraZeneca Pharmaceuticals LP, BeiGene, Janssen Pharmaceutica, and Pharmacyclics. Dr. Zelenetz has disclosed that he has received grant/research support from BeiGene, Gilead Sciences, Inc., MEl Pharma Inc., and Roche Laboratories, Inc.; and consulting fees from Adaptive Biotechnologies Corporation, Amgen Inc., Celgene Corporation, Genentech, Inc./Roche Laboratories, Inc., Gilead Sciences, Inc., Janssen Pharmaceutica Products, LP, Novartis Pharmaceuticals Corporation, and Verastem Oncology; and is a scientific advisor for AbbVie, Inc., AstraZeneca Pharmaceuticals LP, Genentech, Inc., Gilead Sciences, Inc., MorphoSys AG, and Pharmacyclics.

Correspondence: Mazyar Shadman, MD, MPH, Fred Hutchinson Cancer Research Center, 1100 Fairview Avenue N, D5-396, Seattle, WA 98109. Email: mshadman@fredhutch.org; and

Deborah M. Stephens, DO, Huntsman Cancer Institute at the University of Utah, 2000 Circle of Hope RS 5509, Salt Lake City, UT 84112. Email: deborah.stephens@hci.utah.edu

\section{References}

1. Thompson PA, Tam CS, O'Brien S, et al. Fludarabine, cyclophosphamide, and rituximab treatment achieves long-term disease-free survival in IGHVmutated chronic lymphocytic leukemia. Blood 2016;127:303-309.

2. Shanafelt TD, Wang XV, Kay NE, et al. Ibrutinib-rituximab or chemoimmunotherapy for chronic lymphocytic leukemia. N Engl J Med 2019, 381:432-443.

3. Shanafelt TD, Wang $\mathrm{V}$, Kay $\mathrm{NE}$, et al. Ibrutinib and rituximab provides superior clinical outcome compared to FCR in younger patients with chronic lymphocytic leukemia (CLL): extended follow-up from the E1912 Trial [abstract]. Blood 2019;134(Suppl 1):Abstract 33.

4. Woyach JA, Ruppert AS, Heerema NA, et al. Ibrutinib regimens versus chemoimmunotherapy in older patients with untreated CLL. N Engl J Med 2018;379:2517-2528.

5. Fischer K, Al-Sawaf O, Bahlo J, et al. Venetoclax and obinutuzumab in patients with CLL and coexisting conditions. N Engl J Med 2019;380: 2235-2236.

6. Fischer $K$, Ritgen M, Al-Sawaf $O$, et al. Quantitative analysis of minima residual disease (MRD) shows high rates of undetectable MRD after fixedduration chemotherapy-free treatment and serves as surrogate marker for progression-free survival: a prospective analysis of the randomized CLL14 trial [abstract]. Blood 2019;134(Suppl 1):Abstract 36.

7. Davids MS, Brander DM, Kim HT, et al. Ibrutinib plus fludarabine, cyclophosphamide, and rituximab as initial treatment for younger patients with chronic lymphocytic leukaemia: a single-arm, multicenter, phase 2 trial. Lancet Haematol 2019;6:e419-428.

8. Jain N, Thompson PA, Burger JA, et al. Ibrutinib, fludarabine, cyclophosphamide, and obinutuzumab (iFCG) for first-line treatment of patients with CLL with mutated IGHV and without TP53 aberrations [abstract]. Blood 2019;134(Suppl 1):Abstract 357

9. Shanafelt TD, Wang $V$, Kay NE, et al. A randomized phase III study of ibrutinib (PCl-32765)-based therapy vs. standard fludarabine, cyclophosphamide, and rituximab (FCR) chemoimmunotherapy in untreated younger patients with chronic lymphocytic leukemia (CLL): a trial of the ECOG-ACRIN Cancer Research Group (E1912) [abstract]. Blood 2018; 132(Suppl):Abstract LBA-4.

10. O'Brien S, Furman RR, Coutre $S$, et al. Single-agent ibrutinib in treatmentnaïve and relapsed/refractory chronic lymphocytic leukemia: a 5-year experience. Blood 2018;131:1910-1919.

11. Burger JA, Barr PM, Robak T, et al. Long-term efficacy and safety of firstline ibrutinib treatment for patients with CLL/SLL: 5 years of follow-up from the phase 3 RESONATE-2 study. Leukemia 2020;34:787-798.

12. O'Brien S, Hillmen $P$, Coutre $S$, et al. Safety analysis of four randomized controlled studies of ibrutinib in patients with chronic lymphocytic leukemia/small lymphocytic lymphoma or mantle cell lymphoma. Clin Lymphoma Myeloma Leuk 2018;18:803-813.

13. Tam CS, Siddiqi T, Allan JN, et al. Ibrutinib plus venetoclax for first-line treatment of chronic lymphocytic leukemia/small lymphocytic leukemia: results from the MRD cohort of the phase 2 CAPTIVATE study [abstract] Blood 2019;134(Suppl 1):Abstract 35 